\title{
SLFinder, a pipeline for the novel identification of splice-leader sequences: a good enough solution for a complex problem
}

Javier Calvelo ${ }^{1,2,3}$, Hernán Juan ${ }^{1}$, Héctor Musto ${ }^{2}$, Uriel Koziol ${ }^{3}$ and Andrés Iriarte ${ }^{1 *}$ (D)

* Correspondence: airiarteo@gmail. com

${ }^{1}$ Laboratorio de Biología Computacional, Departamento de Desarrollo Biotecnológico, Instituto de Higiene, Facultad de Medicina, Universidad de la República, Montevideo, Uruguay

Full list of author information is available at the end of the article

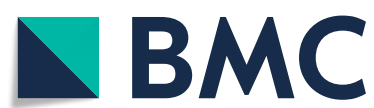

(c) The Author(s) 2020 Open Access This article is licensed under a Creative Commons Attribution 4.0 International License, which permits use, sharing, adaptation, distribution and reproduction in any medium or format, as long as you give appropriate credit to the original author(s) and the source, provide a link to the Creative Commons licence, and indicate if changes were made. The images or other third party material in this article are included in the article's Creative Commons licence, unless indicated otherwise in a credit line to the material. If material is not included in the article's Creative Commons licence and your intended use is not permitted by statutory regulation or exceeds the permitted use, you will need to obtain permission directly from the copyright holder. To view a copy of this licence, visit http://creativecommons.org/licenses/by/4.0/. The Creative Commons Public Domain Dedication waiver (http://creativecommons.org/publicdomain/zero/1.0/) applies to the data made available in this article, unless otherwise stated in a credit line to the data. 
While there is no clear pattern with regards to specific metabolic pathways or functions for the transcripts that are subject to these mechanisms [5-7], SL trans-splicing participates in important regulatory functions such as operon resolution, 5'UTR edition and the incorporation of modified $5^{\prime}$ cap $[1,5,6,8,9]$. At least in some cases, it has been shown that it can also play an important role generating different isoforms by facultative SL trans-splicing (e.g. [10]) or alternative SL trans-splicing acceptor sites [11]. The number of classes of SLs (i.e., Spliced Leaders with a distinct sequence) and the number of copies in each genome varies among different organisms, and at least in some cases, there is evidence of specialization. For example, Caenorhabditis elegans has two distinct types of SLs, one (SL-1) is incorporated at the start of the operon and the other (SL-2) is used to resolve the downstream coding sequences into different transcripts [8]. In the planarian Schmidtea mediterranea it has been described one particular SL that is expressed preferentially on stem cells [12].

The molecular mechanisms involved are poorly understood and are subject of continuous research (e.g. [13]) but evidence indicates that it's closely related to cis-splicing, with several shared regulatory signals $[1,14]$. All identified SL transcripts share a similar secondary structure to the snRNAs (i.e., U1, U2, U4, and U5) that form the spliceosome, suggesting a common evolutionary history $[1,5,14]$. However, its evolution is a topic of debate among researchers, mainly due to the uneven distribution of SL TransSplicing across the phylogeny of eukaryotes $[1,5,14]$.

So far SL Trans-Splicing has been reported in groups such as Euglenozoa [15, 16], Platyhelminthes [17, 18], Nematoda [19, 20], Urochordata [21], Rotifera [22], Cnidaria [23], Dinoflagellata [24], Crustaceans [25] and Amoebozoa [4]. However, it is absent in others such as vertebrates, insects, plants, Fungi and several protists [14, 26]. This brings the question if the mechanism has independently evolved several times (i.e., by modification of cis-splicing) or was present on the eukaryotic last common ancestor and lost many times $[1,5,14,25]$, with the discussion going back and forth as the mechanism is identified in new taxonomic groups (e.g. [25]).

When analyzing a new organism, the first obvious step is the identification of potential SL sequences on the mRNAs. This does not only allow to identify the presence of the mechanism in the group but having these sequences opens the possibility to use methodologies tailored toward SL Trans-Spliced transcripts. For example, "SL Trapping" [27] or "SL-seq" [28], both modified Next Generation Sequencing (NGS) protocols, allow an enriched sequencing of SL trans-spliced transcripts (e.g. [11]). Other approaches exist, but they either focus on identifying trans-splicing acceptor sites on the coding genes (e.g. [29, 30]), then requiring to be experimentally validated and providing no information about the specific SLs involved; or they require known SL sequences [31-34].

Unfortunately, the identification of SL sequences can be a significant roadblock due to technical limitations, specifically the reduced coverage of reads toward the transcript 5 ' end that is typical of poly-A capture [35]. Combined with low or null sequence conservation across different phyla [5], within phylum variability, and several species with multiple SL classes with high nucleotide diversity [31, 36-38], these difficulties make the identification of SL sequences in new species a non-trivial problem. Several authors have tested different approaches to this problem with different degrees of automatization and reliance on previously known information (e.g. [5, 25, 31, 39-44]). 
Nevertheless, currently, there is no standardized protocol or analysis pipeline that allows the identification of putative SL (pSL) sequences, that is why often novel SL sequences are discovered almost by chance (e.g. [31]).

Here we present SLFinder, a four-step pipeline implemented in bash designed to facilitate the identification of novel SL exonic sequences from standard NGS RNAseq data (mRNA enriched by poly-A capture following a non-strand specific protocol). The pipeline first limits the potential candidates and provides a unifying command-line environment where parameters can be quickly adjusted to fit each species and dataset characteristics; while making limited assumptions on the SL sequence and mechanism, namely: 1) the SL sequence is located in the $5^{\prime}$ end of the transcript, 2) the SL sequence is present on the transcripts of many genes, 3) The sequence is not a palindrome, 4) There is at most one copy of it on each transcript, and 5) When mapped to the genome there is a canonical splicing donor site after the $3^{\prime}$ end (GT). In addition, and despite its limitations, the analyses are designed with transcriptome sequences generated using the widely used poly-A capture protocol so it can be applied to a larger group of organisms.

In order to evaluate SLFinder, we analyzed RNAseq data from several species with and without known SL Trans-Splicing and compared our predictions with the reported sequences in the bibliography. To better represent the intended use of the software on the identification of novel SL sequences, no manual intervention was carried out to curate the results (contrary to our recommendations when using this software).

\section{Implementation}

Mandatory input data

For these analyses three inputs are necessary: 1) one or more assembled transcriptomes from the species of interest, following a de novo approach with Trinity $[45,46]$; 2) a reference genome from the species and 3) an external database with Protein or cDNA (ideally from the same species or a reliable database such as SwissProt from Uniprot) for loci annotation.

Trinity can be replaced as the assembler following the instructions included on the manual, however, it is important to conduct an entirely de novo strategy to ensure that reads containing the SL sequence are not excluded of the final transcript. In addition, while we didn't thoroughly test its effect, read normalization based on kmer frequency (e.g. [46]) is discouraged since reads from multiple transcripts will have the SL sequence and could potentially be partially discarded in some datasets. The longer the species SL sequences, the greater this issue is expected to be.

\section{Strategy}

Basically, the pipeline recovers potential SL exonic 3' regions by looking for frequent kmers on the transcripts ends, extends them as much as possible by attempting to assemble them in contigs, and then filters out likely false positives based on sequence orientation, abundance, genomic data and overlap with annotation to known proteins. In practice, however, there are two issues to solve to implement such a straightforward approach. First, false positives due to biased kmer counts, which can be a result of the reconstruction of more than one isoform for a gene and other biological factors such as 
very similar transcripts from different genes of the same multigenic family. Second, the loss of strand information during sequencing in standard RNAseq sequencing, so that each transcript can be assembled either as the sense strand or as its reverse complement. Both main issues are addressed by our pipeline.

The pipeline overview is presented on Fig. 1. First, the redundancy in the de novo assembly transcriptome is reduced in SLFinder-Step0, hereafter referred to as Step0, by retrieving the $5^{\prime}$ and $3^{\prime}$ ends of the longest isoforms of each gene. Isoforms of the same gene are identified based on Trinity's contigs name convention. Alternative strategies can be implemented (e.g. clustering based on sequence identity) following the manual instructions. Regardless of the chosen method, once redundant sequences are filtered the next step is to identify SLs among the more commonly observed sequences in the transcripts ends. In an ideal situation, the SL sequence should be located at the exact

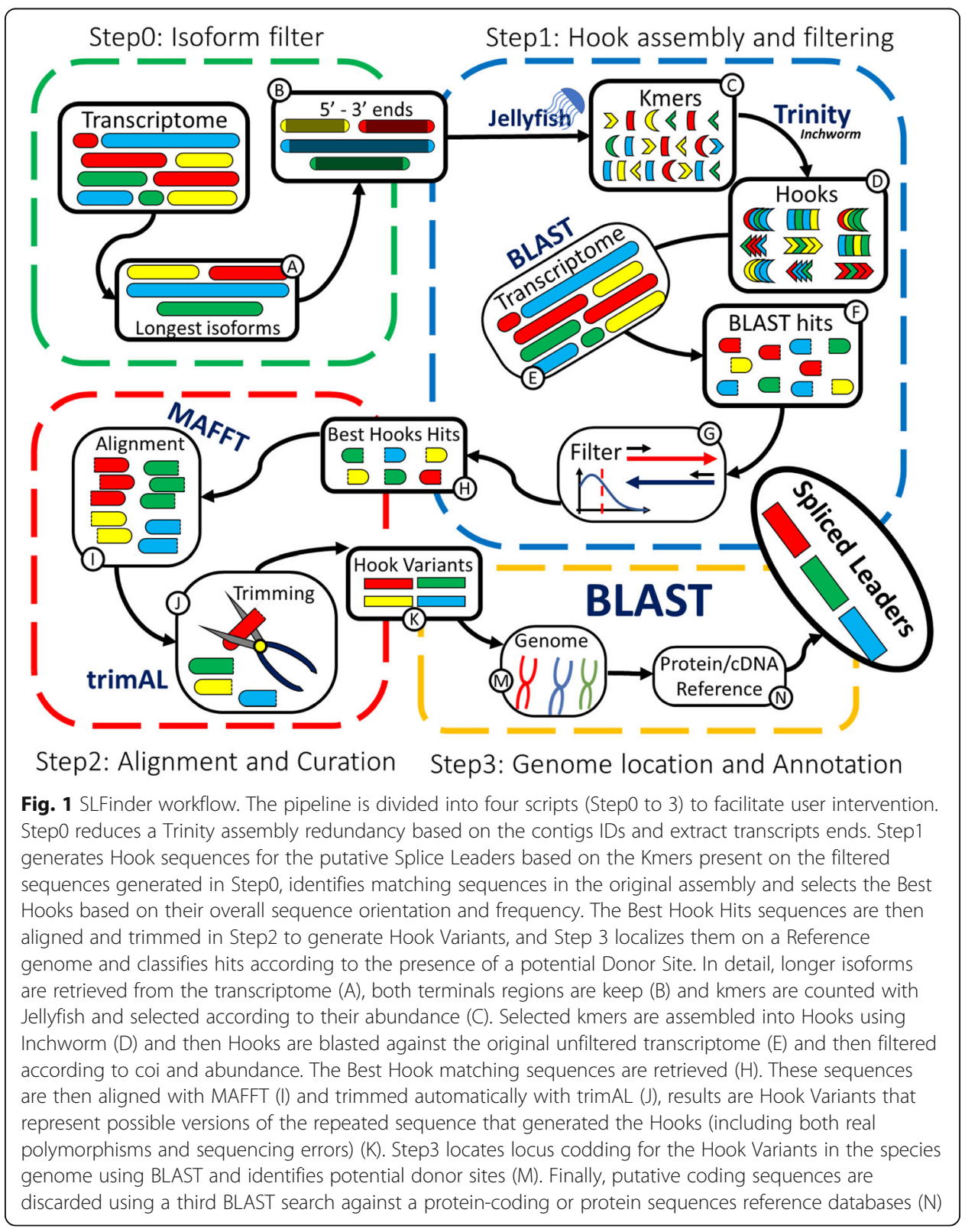


beginning of the assembled transcripts and cleaner results should be obtainable by retrieving from the transcript end a fragment similar in size to the expected length of the SL. However, we noticed that the assemblies used to validate this pipeline often presented non-conserved, mainly low-quality, sequences that preceded the known SL sequence. Instead, Step1 achieves this by counting the kmers present on the filtered sequences (and their reverse complement) with Jellyfish software [47]. Those kmers observed below a given threshold are discarded (by default $0.0005 \%$ of the total contigs after filtering, in practice $\approx 10$ contigs, depending on the dataset), and then assembled in longer sequences with Inchworm, Trinity's first module. The resulting sequences, hereafter referred to as "Hooks", are a collection of true SL sequences (if present) and every other common sequence found on the filtered transcriptome.

To further narrow down candidates, Step1 analyzes the location and orientation of matching sequences in the original transcriptome. A Blast search [48] is conducted to "fish out" similar sequences among the transcriptomic contigs (with the "-task blastnshort" option and "-evalue 1e-2"). Hooks are selected according to their number of hits and sequence orientation in the assembled transcriptomes. Since we are working without information on the strand, the transcripts can be assembled either sense or antisense; and so, can be the Hooks that are generated from these transcripts. However, if our assumption 3 holds (i.e., the sequence is not a palindrome) and the Hook represents a true SL (meaning that it is located at the $5^{\prime}$ end on the transcript) its hits on the transcriptome should be found in two mutually exclusive configurations, depending on the orientation of the Hook: 1) forward-oriented at the Start of a sense assembled transcript or reverse oriented at the End in an anti-sense one if the Hook was generated in a sense configuration (named Sf and Er orientations, respectively); or 2) reverse oriented at the start of a sense assembled transcript and Forward oriented at the end of an anti-sense one (named Sr and Ef orientations, respectively) (Fig. 2a).

With this in mind, we created a simple consistency orientation index (coi) to evaluate each potential Hook (Fig. 2). A coi equal to 1 means that the Hook's hits are all oriented in one and only one of the valid configurations. Testing, however, shows Hooks for known SLs can have some hits that do not follow these rules but their coi is always high (i.e., above 0.95; see Results). In addition, tests show that Hooks with few hits on the transcriptome often have a high coi by chance, even when they are not SL sequences. To compensate we also introduce an Observation Count Cutoff (occ) filter that is simply the median of all identified Hooks. Finally, transcripts with multiple hits for the same Hook are excluded and reported separately for user inspection. These transcripts may represent chimeric sequences generated during the assembly process $[49,50]$ or short repeated sequences. Hooks that pass these filters are selected for further analysis.

The sequences of these selected Hooks, hereafter referred to as Best Hooks, are retrieved for further analysis. The transcript's ends with a BLAST match (with the "-task blastn-short" option and "-evalue 1e-2") to each Best Hook are retrieved (from the transcript end until two bases after the match end in order to recover as much sequence from the pSL sequence).

The next filtering step in the pipeline consists of locating and analyzing genomic loci matching the Best Hooks from which they are potentially transcribed (putative SL genes). However, first, it is necessary to address three issues: high redundancy, noisy 


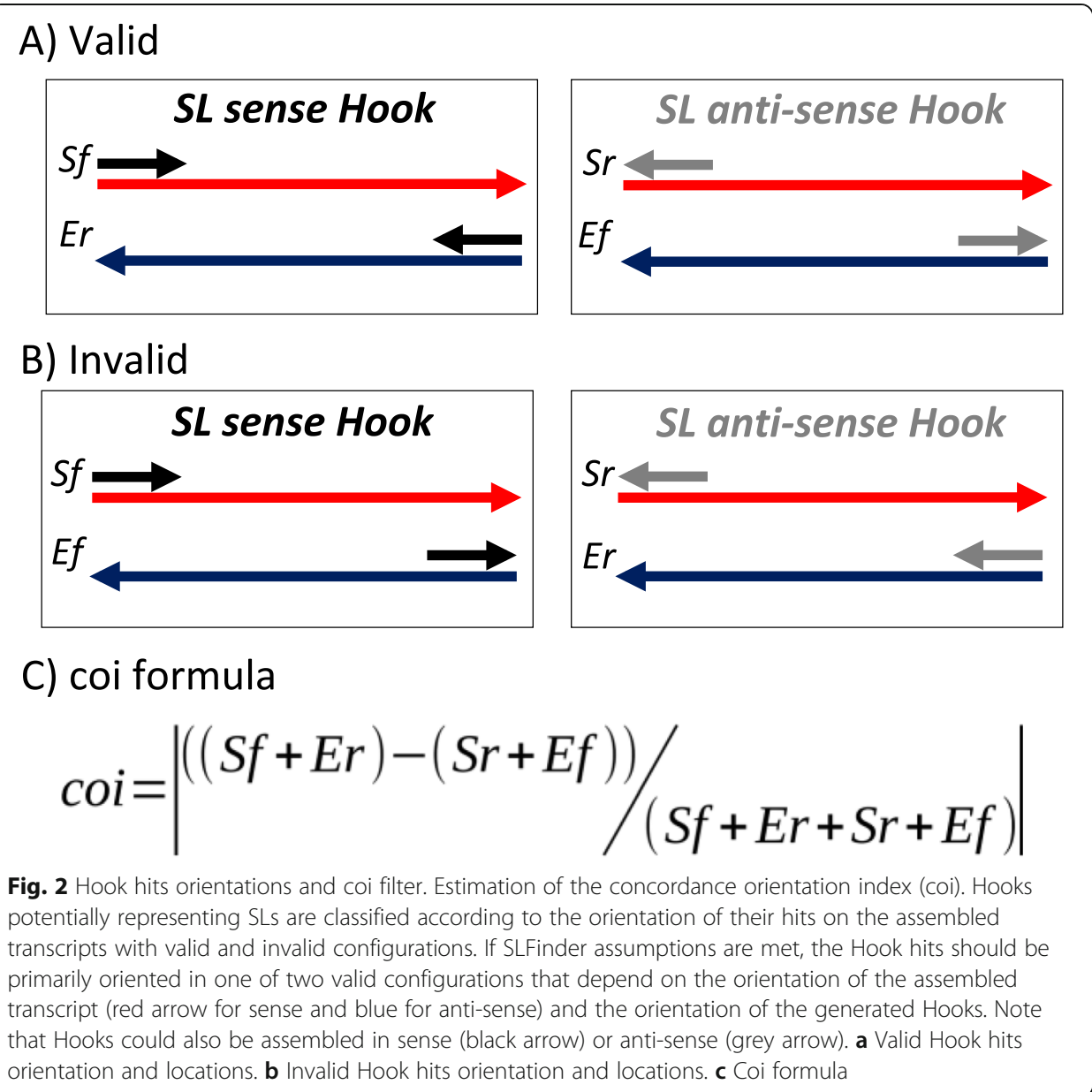

sequences coming from sequencing and assembly errors, and imprecise pSL delimitation. Without knowing the SL sequence there is no reliable way to address these problems with a one-base precision, nevertheless, Step2 minimizes them by first clustering all sequences according to sequence identity with CD-HIT-EST [51] with a $100 \%$ identity threshold by default, followed by alignment with MAFFT [52] using the accuracy oriented method G-INS-I “--globalpair --maxiterate”. Finally, sequences are automatically trimmed with trimAL [53]. The resulting sequences, referred to as "Hook Variants", represent possible versions of the repeated sequence that generated the Hook (including both real polymorphisms and sequencing errors). Depending on the data, it might be necessary to re-run this step several times with different parameters or even manually curate the sequences before continuing with Step3 (see the software manual for detailed instructions). To facilitate this process, Step2 also generates sequence logos before and after trimming with Weblogos3 [54].

Step3 carries out a BLAST (-task blastn-short) search of the Hook Variants against the provided reference genome to identified pSL coding loci. Since some level of noise is expected in the Hook Variants sequences, even when they represent true SL (see below), the BLAST search is configured with a 100\% identity threshold, ungapped, and a high query coverage ( $90 \%$ by default). In practice, these thresholds allow mismatches in the terminal region of the Hook Variant. Once identified, Step3 searches for the 
existence of a potential donor site and attempts to annotate the region with an external CDS or protein reference with either blastn or tblastx. As a final fail-safe to check the inaccuracy in the pSL delimitation, Step3 takes the following considerations when reporting a potential donor site: 1) It analyses 4 bp surrounding each Hook Variant 3' end hit in the genome (excluding mismatches in the extremes) looking for a possible splice donor site ("GT"). If one "GT" is found, step3 reports either "5prima" or "3prima" depending on the hit orientation, simplified to "Clear donor site" in this paper. 2) If the longest matching Hook Variant with a donor site overlaps with possible splice donor site (i.e., the sequence ends with a "G" or "GT" that matches with the splice donor site) an "*" is included in the report to indicate that manual inspection is advised. 3) If a potential donor site is found in fewer than $80 \%$ of Hook Variants matching a locus, the site is reported as "Unclear". Finally, a BLAST search between the region surrounding each locus and the provided Protein/cDNA reference dataset is conducted, and loci with matches are discarded. In every step the user can check the Hook Variants and blast results to reconsider or inspect some discarded Hooks.

A putative SL coding Locus was considered valid if a potential donor site was identified and there were no known protein-coding sequences located close to the locus (by default 100pb, this parameter can be changed by the user). Sequences for loci with and without a clear donor site are clustered with CD-HIT-EST in pSL sequences (100\% identity Threshold). The final output also includes multiple sequence alignment of each locus done with MAFFT (G-INS-I “--globalpair --maxiterate") and its original Hook Variants to facilitate manual inspection.

\section{Test data}

Test data was selected from species according to known presence or absence of SL Trans-splicing, the existence of a reference genome and availability of RNAseq following a poly-A capture protocol. The final species list comprised Aplysina aerophoba, $C$. elegans [19], Ciona intestinalis [21], Drosophila melanogaster, Hydra vulgaris [23], Mus musculus, Saccostrea glomerata, and Schistosoma mansoni [18]. Schistosoma mansoni, has been reported to have a single SL class with a long sequence (36 bp), represents an ideal scenario to test SLFinder. Meanwhile, $C$. intestinalis with single short SL (16 pb) allows investigating how the pipeline behaves with shorter sequences. Finally, C. elegans and $H$. vulgaris have multiple SL sequences (some of them with known sequence diversity among their coding SL-RNAs, e.g. SL2 in C. elegans) which will test SLFinder ability to identify and retrieve different SLs when present.

Transcriptomic and genomic data used on these analyses are detailed in Table 1. Non-control samples from experimental studies (i.e. response to pathogens or other stimulus) were discarded. Genomic locus annotation was carried out with the SwissProt database from Uniprot (Downloaded 01/05/2019). In addition, since several genome assemblies are available for S. mansoni; mostly based on Protasio et al. 2012 work [55] but improved and annotated following different methodologies for their curation and annotation; SLFinder was tested using 2 reference genomes: one from Wormbase Parasite (WBPS) improved with PacBio data and one from GeneDB that is more fragmented but with several SL-RNA genes annotated. 
Table 1 Datasets utilized to validate and evaluate SLFinder

\begin{tabular}{|c|c|c|c|c|}
\hline Species & Taxon & RNAseq BioProject & Ref. Genome Assembly & Reported SLS \\
\hline A. aerophoba & Porifera & PRJEB26562 & GCA_900275595.1 & No \\
\hline C. elegans & Nematoda & PRJNA270896 & PRJNA13758 ${ }^{\mathrm{b}}$ & Yes \\
\hline C. intestinalis & Urochordata & PRJNA396771 & GCF_000224145.3 $3^{\mathrm{a}}$ & Yes \\
\hline D. melanogaster & Insecta & PRJNA318586 & GCF_000001215.4 & No \\
\hline H. vulgaris & Cnidaria & PRJNA497966 & $\mathrm{Hm} 105^{\mathrm{c}}$ & Yes \\
\hline M. musculus & Vertebrata & PRJNA319673 & GCF_000001635.26 & No \\
\hline S. glomerata & Molusca & PRJNA487836 & GCA_003671525.1 & No \\
\hline S. masoni & Plathelminthes & PRJNA225599 & PRJEA36577 $7^{\mathrm{b}}$ and GeneDB & Yes \\
\hline
\end{tabular}

a Available on NCBI database

${ }^{\mathrm{b}}$ Available on WBPS database

c Hydra 2.0 Genome Project

Read quality for RNAseq data was assessed with FastQC [56] and low quality bases along with adapter sequences were removed with Trimmomatic v0.36 [57] (options: ILLUMINACLIP: TruSeq3-PE.fa:2:30:10, SLIDINGWINDOW: 5:20 and MINLEN: 50). Transcriptomes were de novo assembled with Trinity v2.8.3, without read normalization.

\section{Bioinformatic analysis and pipeline evaluation}

Analyses were carried out in a desktop computer with 96Gb of RAM and 32 threads/ 16 cores (only 4 threads were used on each run). Program versions used are listed on Table 2 with default parameters (with exception of C. intestinalis). Pipeline accuracy was tested by sequence comparisons with known SL sequences (Additional file 1), verifying the match of the predicted SL locus with the annotated SL within $100 \mathrm{bp}$ range. This comparison was done using gffread [58]. In addition, each potential locus was manually inspected, and "seqkit locate" was utilized to verify the transcripts carrying specific pSL sequences in order to detect and categorize artefacts. Figures of sequence alignments were generated with BioEdit v7.0.5.3 [59].

\section{Results}

A total of 32 transcriptomes ( 9 from A. aerophoba, 6 from C. elegans, 3 from C. intestinalis, 4 from $D$. melanogaster, 5 from $H$. vulgaris, 2 from M. musculus, 1 from $S$.

Table 2 List of programs and software packages utilized by SLFinder, including the version utilized in this paper and the basic tasks they carry out

\begin{tabular}{lll}
\hline Program & version & Tasks \\
\hline Blast & v2.6.0 & $\begin{array}{l}\text { Sequence searches against Transcriptome assemblies, Genome and Protein reference } \\
\text { database. }\end{array}$ \\
cd-hit-est & v4.7 & Sequence clustering to simplify results and reduce runtimes \\
Jellifish & v2.2.6 & Kmer counts \\
MAFFT & $v 7.307$ & Sequence Alignment \\
Seqkit & v0.10.0 & Basic sequence manipulation \\
trimAl & v1.2rev59 & Hook Variant generation by automatic trimming \\
Trinity & v2.8.3 & Hook assembly from Kmers \\
Weblogos & v3.6.0 & Sequence Logos generation to facilitate manual curation \\
\hline
\end{tabular}


glomerate, and 2 S. mansoni) were assembled and analyzed (Basic descriptor metrics are shown in Additional file 2). Running times per step where highly dependent on the dataset (Table 3) mainly depending on the number of reads to process. No Hook sequence passed the coi filter in Step1 for the species without known SLs A. aerophoba, D. melanogaster, M. musculus and S. glomerata (Additional file 3).

Positive results were identified for the species C. elegans, Hydra vulgaris and S. mansoni, all with previously described SL. SLFinder also identified the SL reported for $C$. intestinalis after changing the parameters to account for short SLs (15-base kmer length, 14 Inchworm assembly kmer, and no filtering according to the median count value).

In the following sections we will describe the results obtained by each Step of SLFinder (Step 1 Hook generation and filtering, Step 2 Hook Variant trimming, and Step 3 putative SL (pSL) loci identification) on each positive dataset. Since the intent of this software is novel SL identification, we will focus on features of SLFinder reports that depart from the true SL sequence (e.g. longer/shorter sequences than expected and potential false positives results).

\section{Caenorhabditis elegans dataset}

A total of 13 hooks were generated in Step1, three of which passed both the coi and the occ filters and resulted in 246 different Hook Variants after Step2. Comparison with known SL sequences showed that the Best Hooks "a1-9915" and "a11-1448" corresponded to the known SL-1, while "a10-178" to SL-2 (Fig. 3a).

Step3 identified 26 putative pSL loci in the reference genome, 18 of which were previously reported as SL-RNA genes in the genome annotation (Additional file 4). Thirteen pSL were reported as having a clear potential donor site and were later clustered into 8 sequences; hereafter referred to as Celegans_pSL-(1 to 8). Another 10 loci were reported as Unclear due to the presence of several bases in the $3^{\prime}$ region in several variants for the Hook "a1-9915" that overlapped with the splice donor site (Additional file 5). Most of these loci were located on Chromosome $\mathrm{V}$ in a cluster of $\approx 13 \mathrm{~kb}$ and were grouped into a single sequence identical to Celegans_pSL-7. In addition, Locus-5 and -26 were reported without a donor site and Locus- 25 was not analyzed because SLFinder failed to determine its orientation due to low count numbers in the transcriptome. Manual inspection showed that both Locus- 25 and Locus- 26 have a potential donor site masked by a three base extension in the $3^{\prime}$ end (GTA) of the only

Table 3 SLFinder steps performance for all datasets

\begin{tabular}{|c|c|c|c|c|c|}
\hline Data Set & Step0 & Step1 & Step2 & Step3 & Total \\
\hline A. aerophoba & $32 \mathrm{~m} \mathrm{12s}$ & $0 \mathrm{~m} \mathrm{34s}$ & $x$ & $x$ & $32 \mathrm{~m} \mathrm{46s}$ \\
\hline C. elegans & $4 \mathrm{~m} 23 \mathrm{~s}$ & $0 \mathrm{~m} 24 \mathrm{~s}$ & $13 \mathrm{~m} 25 \mathrm{~s}$ & $1 \mathrm{~m} 08 \mathrm{~s}$ & $19 \mathrm{~m} 46 \mathrm{~s}$ \\
\hline C. intestinalis & $4 \mathrm{~m} 29 \mathrm{~s}$ & $1 \mathrm{~m} 54 \mathrm{~s}$ & $0 \mathrm{~m} 03 \mathrm{~s}$ & $0 \mathrm{~m} 43 \mathrm{~s}$ & $7 \mathrm{~m} \mathrm{09s}$ \\
\hline D. melanogaster & $5 \mathrm{~m} 36 \mathrm{~s}$ & $0 \mathrm{~m} \mathrm{20s}$ & $x$ & $x$ & $5 \mathrm{~m} 56 \mathrm{~s}$ \\
\hline H. vulgaris & $15 \mathrm{~m} \mathrm{17s}$ & $57 \mathrm{~m} \mathrm{26s}$ & $19 \mathrm{~m} 58 \mathrm{~s}$ & $14 \mathrm{~m} 35 \mathrm{~s}$ & $1 \mathrm{~h} 47 \mathrm{~m} \mathrm{16s}$ \\
\hline M. musculus & $7 \mathrm{~m} 14 \mathrm{~s}$ & $2 \mathrm{~m} \mathrm{10s}$ & $x$ & $x$ & $7 \mathrm{~m} 24 \mathrm{~s}$ \\
\hline S. glomerata & $24 \mathrm{~m} 24 \mathrm{~s}$ & $0 \mathrm{~m} 25 \mathrm{~s}$ & $x$ & $x$ & $24 \mathrm{~m} 59 \mathrm{~s}$ \\
\hline S. mansoni & $6 \mathrm{~m} 53 \mathrm{~s}$ & $0 \mathrm{~m} \mathrm{38s}$ & $0 \mathrm{~m} 06 \mathrm{~s}$ & $3 \mathrm{~m} 03 \mathrm{~s}$ & $10 \mathrm{~m} \mathrm{40s}$ \\
\hline
\end{tabular}




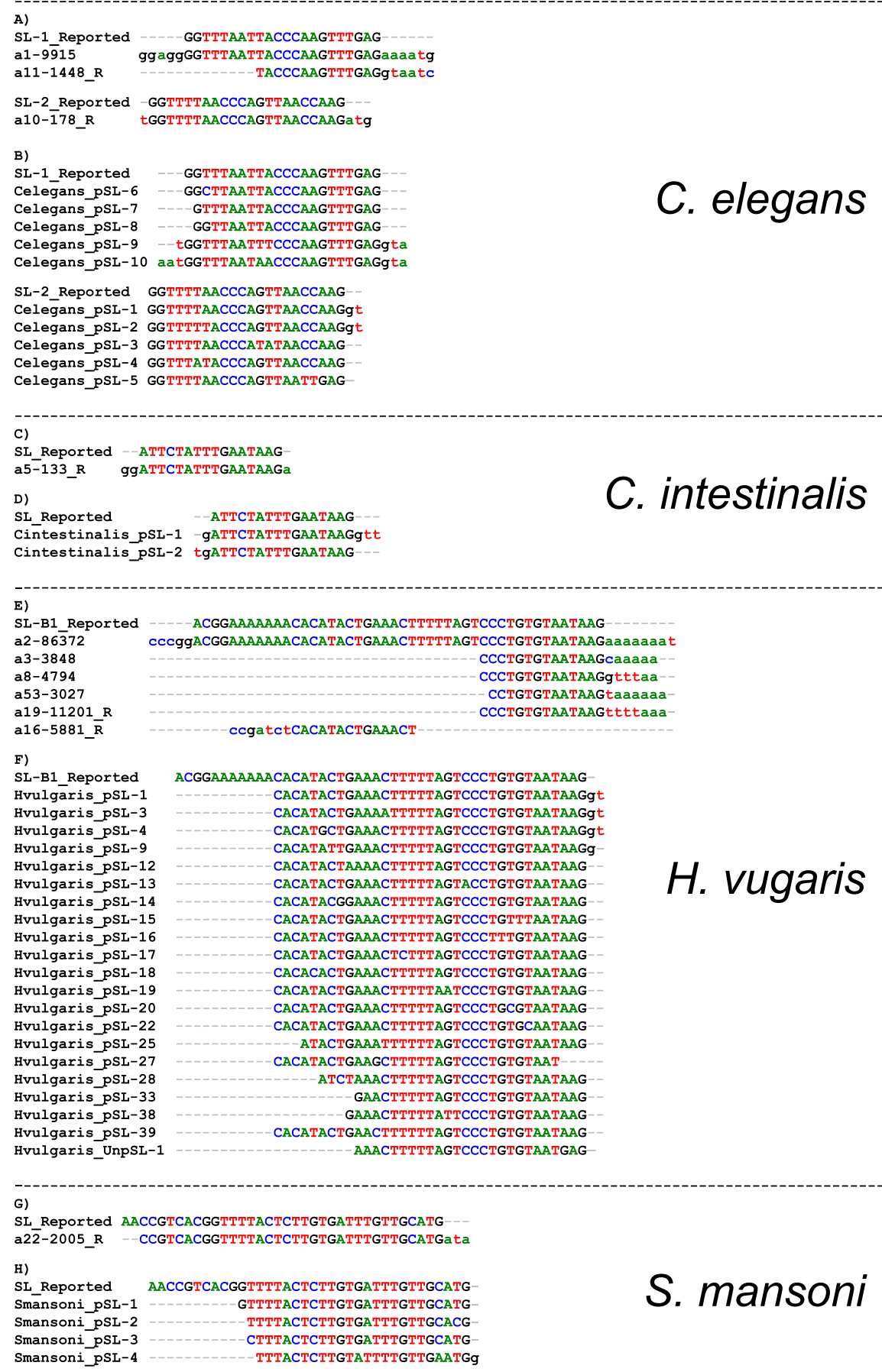

\section{C. elegans}

G)

SL Reported AACCGTCACGGTTTTTACTCTTGTGATTTGTTGCATGa2-2005_R - CCGTCACGGTTTTACTCTTGTGATTTGTTGCATGata

H)

SL Reported AACCGTCACGGTTTTACTCTTGTGATTTGTTGCATG SL_Reported AACCGTCACGGTTTTACTCTTGTGATTTGTTGCATG Smansoni_pSL-1
Smansoni pSL-2 Smansoni_pSL-2 Smansoni_PSL-4 _-_._TTACTCTTGTATTTTGTTGAATG

Fig. 3 SLFinder predictions for all datasets. SLFinder summary results and known SL sequences reported in analyzed species. For each species, the figure shows selected Best Hooks found in Step 1 (a, c, e, and $\mathbf{g}$ ), and predicted putative SLs (pSL) after Steps 2 and $3(\mathbf{b}, \mathbf{d}$, f, and $\mathbf{h})$. "_R" referred to reverse complement sequence of the Best Hook found. Figures of sequence alignments were generated with BioEdit v7.0.5.3 [59]

matching Hook Variant for each, hereafter referred to as Celegans_pSL-9 and -10, respectively (see similar cases in Additional file 5).

Potential Spliced Leaders Celegans_pSL-6, - 7, - 8, - 9, and - 10 match the previously described SL-1 and their nucleotide differences were limited to the $5^{\prime}$ region, whereas 
Celegans_pSL-1, $-2,-3,-4$ and -5 represent different variants of SL-2 and are slightly more diverse in their nucleotide sequences (Fig. 3b). These observations are in concordance with the genome annotation and previous results for C. elegans [2].

Site-specific observations of these loci are included in Additional file 4. Of particular relevancy are Locus-12 and -20, both display partial repetitions of SL-1 following the reported hit (Additional file 6). Verification of the functionality of these SLs loci is beyond the scope of this paper and the capabilities of SLFinder, a pattern search against the reads only identified six read pairs bearing Locus-12 repeat across all samples.

In Summary, SLFinder identified both SL classes, SL-1 and SL-2, previously reported for C. elegans and located several of their described SL-RNAs loci (10 for SL-1 and 8 for SL-2), in addition to five not previously annotated copies of SL. While verifying the functionality of these new SL-RNAs is beyond the scope of this paper, our results suggest that at least two of them (Locus-12 and -20) are presumed to be pseudogenes due to the presence of fragments from SL-1 following the 3' end.

\section{Ciona intestinalis dataset}

Using the modified parameters (15-base kmer length, 14-base Inchworm assembly kmer and removing occ filtering), Step1 generated 81 Hooks but only "a5-133" passed the coi filter. Twenty-three Hook Variants were identified for "a5-133" in Step2. Results show that the Hook matches the sequence of the previously reported SL [21] (Fig. 3c).

Step3 identified 38 putative loci in the genome, 23 of which have a predicted protein-coding gene in the matched region according to the available annotation (Additional file 7). Long non-coding RNA (lncRNA) are annotated surrounding Locus-8, -12, and -15 in this dataset (XR_717275.3, XR_003396022.1 and XR_ 003396339.1 respectively), but their functions are unknown and only Locus-12 is encompassed by the lncRNA included by its hit. Fourteen pSL were reported with a clear potential donor site and were grouped into two clusters; hereafter named as Cintestinalis_pSL-1 and -2; that differ on their extension toward both sequence ends (Fig. 3d). The former shows a 3' extension "GTT" which overextends the expected donor site for the SL and ends next to another "GT" in the genome. Detailed observations are included in Additional file 7.

Despite its shorter size, once the software parameters were properly fine-tuned, SLFinder was able to recover the reported SL sequence for this species. However, the results are not as clear as other datasets analyzed, indicating that these conditions are near the limits of what is possible to obtain with this strategy.

\section{Hydra vulgaris dataset}

For this species Step 1 generated 31 Hooks, 6 of which passed both coi and frequency filters and their matches in the transcriptome were processed on 385 Hook Variants. Comparison with known SL sequences for the species shows that the longest Hook "a2-86,372" matches SL-B1 (reported in [36]); while hooks "a3-3848", "a8-4794", "a16-5881", "a19-11,201" and "a53-3028" match only the terminal region (Fig. 3e). 
Unfortunately, the $5^{\prime}$ region of the observed Hook Variants, from 16 to 32 bases, was lost in Step2 during trimming (Additional file 5d).

Step3 identified 239 loci in the genome many of which were found in close proximity to annotated protein-coding regions (Additional file 8). 93 loci were reported with a clear donor site ("Clear") and 59 with an unclear donor site ("Unclear"). The former was clustered in 37 pSL sequences, Hvulgaris_pSL-(1 to 37), and the latter in 10, Hvulgaris_UnpSL-(1 to 10). Hvulgaris_UnpSL-4 has an identical sequence to Hvulgaris_ pSL-1, Hvulgaris_UnpSL-3 to Hvulgaris_pSL-6, and Hvulgaris_UnpSL-7 to Hvulgaris pSL-9 (Additional file 9). In addition, 5 loci were not analyzed because SLFinder failed to determinate their orientation due to low counts in the transcriptome. Among these, Locus-46 and -112 display a potential donor site and are included in the further discussion as Hvulgaris_pSL-38 and -39 respectively.

The manual inspection revealed several issues that suggest they are most likely non-functional versions of SLs that guarantee further analysis. For the purposes of presenting the tool, however, they were considered non-functional. Removing them reduces the pSL unique sequences to 21 (Fig. 3f) (see the full set of pSLs generated by SLFinder in Additional file 9 and detailed observations in Additional file 8). Note that many pSL loci displayed a donor site that overlaps with the known last base of the SL (as previously described for C. elegans), while others presented extensions that led to an alternative "GT" (as previously described for C. intestinalis) without including the expected donor site. While is possible that the latter pSLs represent longer than already reported SL sequences, testing this will require additional studies that are beyond the scope of this paper. Furthermore, an inspection of the transcripts bearing these sequences indicates that these pSL loci match some transcripts for several bp after the pSL sequence (Data not shown) raising further doubts on their functionality. Lastly, 28 loci showed partial repeats of SL sequences, including some of the previously reported SLs (SL-D, -F, and -G) that were not recovered by SLFinder (Additional file 6).

In summary, $H$. vulgaris was the most complex dataset analyzed, with several potential pseudogenes for the SL-RNAs identified. This is, likely in no small extent, related to their complex evolutionary history [36]. Unfortunately, SLFinder failed to identify the other 6 SL reported for the species [36]. A pattern search with seqkit locate of the terminal region of these SLs on the original fastq files indicates a marginal presence of SL-B2, SL-B3, SL-B4, SL-D and SL-G in the dataset, so the most probable cause of this false negatives is their low prevalence in the analyzed RNAseq data (Data not shown).

\section{Schistosoma mansoni dataset}

One Hook ("a22-2005") out of 30 generated in Step1 passed both coi and frequency filters and was then processed into 11 Hook Variants by Step2. Comparison with the known SL sequence for S. mansoni shows that this Hook represents the reverse complement of the described SL in almost its entirety (Fig. 3g). As with the H. vulgaris dataset, part of the $5^{\prime}$ region of the Hook that was recovered was lost during trimming in Step2 due to the poor alignment quality of this region. This could be at least partially explained by missing information and high variability among the retrieved sequences in the transcriptome assemblies (Data not shown). 
When using the WBPS reference genome, Step3 identified 132 pSL loci, only 13 in the proximity to protein-coding genes (Additional file 10). Most of them showed a clear donor site and were clustered in 3 groups; hereafter referred to as Smansoni_ pSL-(1 to 3$)$. The remaining 9 loci were reported as lacking a potential donor site. This was confirmed by manual inspection in all cases except for Locus-128, in which the donor site was masked by the retention of $3 \mathrm{bp}$ on the $3^{\prime}$ end of the generated Hook Variant; hereafter referred to as Smansoni_pSL-4. All four pSL are shown in Fig. 3h while loci coordinates and observations are reported in Additional file 10. Note that only Smansoni_pSL-1 was encoded by several loci. On the other hand, Smansoni_pSL-2 had a substitution in the terminal ATG of the SL. This ATG was reported as completely conserved in all studied Platyhelminthes (see [5]). A pattern search of the terminal region of this pSL reveals a marginal presence on the reads from both sequenced samples, indicating very low expression of this SL variant in the dataset (Data not shown).

Surprisingly, only $22 \mathrm{pSL}$ loci were identified when using the GeneDB reference genome (Additional file 11). Fifteen of these presented a clear potential donor site and were clustered in the same three pSL classes found using the WBPS reference genome (see above), including Smansoni_pSL-4 (Data not shown). Five pSL coding loci were already reported as SL-RNA coding genes, including one locus that was reported without a donor site because of missing information in the reference genome.

In the case of S. mansoni, SLFinder identified the known SLs, including one possible pseudogene, with the only drawback of a partial recovery of its 5 ' region. Results also show the importance of the Reference Genome, as illustrated by the number of pSL loci found in the assemblies of WBPS and GeneDB.

\section{Discussion}

\section{Considerations when using SLFinder}

The strategy presented here, although effective, has shortcomings that originate from the input data and the minimal assumptions regarding the SL sequences. SLFinder requires enough SL exon sequences to be present in the de novo transcriptome assembly. This may be an important issue when considering the widely distributed poly-A enrichment strategy for RNAseq in eukaryotes, nevertheless, our results clearly show that identifying SL sequences and loci is possible in real datasets. Short SL sequences, poor data quality, and the inappropriate reference genome, or a combination of the three may also be issues to consider. See for instance the results of $C$. intestinalis dataset, which could be handled however with specific parameters settings. When dealing with such cases we recommend changing kmer size, ideally using similar organisms a guideline, and annotate every hit for a Hook with a high coi value. Bear in mind that because of these limitations, negative results should not be considered evidence of absence of SL Trans-Splicing.

In addition, the lack of reliance on known SL sequences combined with the approach taken to generate Hook Variants are the source of the issues in identifying the donor site described in Results. Basically, the problem is how to answer the question: "Where does the sequence end when the sequence is unknown?". SLFinder solves this issue by trimming according to alignment quality and then localizing them in the reference 
genome for further pinpointing the SL extension. While most of the issues with automatic trimming (see [60]) don't apply in this context, a side effect of this strategy is the addition of non-SL bp if they are present in enough transcripts, along with a common loss of the SL 5' region during the trimming of Hook hits (both observed in the Results section). Nevertheless, both drawbacks can be properly addressed with an informed user intervention that is facilitated by SLFinder modularity, either by adjusting trimAL parameters or manually processing the alignments (Note that these modifications might affect Step3 results as some divergent pSL Loci will be lost).

The quality of the reference genome and other biological features of the species play an important role in SLFinder accuracy and performance. As stated before, the reference genome is a key piece of information when pinpointing the PSL sequence and filtering out Hook Variants generated due to sequencing and/or assembly errors. This is clearly shown in the analyses of $H$. vulgaris and S. mansoni datasets. On the one hand, SL prediction in $H$. vulgaris was far from straightforward given the high abundance of pSL coding loci found, many of which are likely false positives. This result may be explained, at least in part, by the high prevalence of transposable elements in their genome [36]. In the case of S. mansoni the differences observed between WBPS and GeneDB genome assemblies may explain the different results obtained with SLFinder for this species. A better assembly may help identify more SL loci, as is the case of WBPS assembly. Note that PacBio technology was used to improve assembly quality in this assembly [61].

In the absence of a reference genome, the Hook sequences generated during Step1 and the Hook Variants in Step2 offer a good alternative, but it would require validation based on homology (SL sequences from other closely related taxa) or wet lab experimental approaches.

\section{Advantages of SLFinder}

Taxon sampling bias has been a constant issue in the study of SL trans-splicing across the tree of life. For example, Bitar et al. study conducted a study based on BLAST searches against public databases and identified mostly SL-1 like sequences in the phylum Nematoda. Results included species like Globodera rostochiensis that possess known divergent SL sequences [38] and Heterodera glycines for which more SL classes were latter described [31]. SLFinder represents a solution to this problem by providing a straightforward method to identify pSL sequences that is not based on sequence homology.

The use of over-represented kmers to identify regulatory regions is not a new approach for exploratory analysis of DNA sequences [62] and was applied to identify SL sequences before [36]. However, the novel but simple filters implemented in SLFinder allowed the easy recovery of known SL exonic sequences of the four species with this splicing mechanism in just a few hours; and in the case of C. elegans and S. mansoni even identifying the known SL-RNA coding loci. Only the $C$. intestinalis dataset required a fine-tuning of SLFinder parameters to account for a shorter than expected SL sequences.

Potential SLs sequences identified with this pipeline can be validated through experimental procedures like RT-PCR or 5' RACE (e.g. [31, 37]) or can be used as input data 
for other informatics analyses like the ones implemented by SLQuant [34] and, UTRme [33]. Even a simple pattern search (e.g. [31]) could be used to identify the acceptor genes in order to further analyze mRNA maturation in the species of interest. The identified putative SLs coding loci can be used to further validate the SL-RNA by looking for the sm site or the RNA secondary structure $[1,5,37]$.

\section{Conclusion}

SLFinder offers a practical alternative for the discovery of novel SL sequences aside from homology searches or fortuitous identification. This modular pipeline was proved with freely available RNAseq data for organisms with and without reported Splice Leader sequences with very good results. Putative SLs found by SLFinder can be later refined regarding their exact length and confirmed through additional bioinformatics analyses and wet lab experiments. This software represents a step forward toward a more comprehensive understanding of the distribution of SL Trans-Splicing in the tree of life, its evolutionary history and importance.

\section{Supplementary information}

Supplementary information accompanies this paper at https://doi.org/10.1186/s12859-020-03610-6.

Additional file 1: Supplementary Table 1. Reported SL sequences for the species C. elegans, C. intestinalis, $H$. vulgaris and S. mansoni.

Additional file 2: Supplementary Table 2. Basic descriptors of the analyzed datasets. Data Set, GenBank SRA ID, $N^{\circ}$ Reads, N Trimmed Reads, Assembled Bases, Total Transcripts, N50, Median Contig Length, Average Contig Length, Genes predicted by Trinity (Trinity Genes) and GC content.

Additional file 3: Supplementary Table 3. Hook hits obtained for each dataset (Step 1 - F in Fig. 1). Hit counts according to position and orientation in the contig, coi score and observation count cutoff (occ) are indicated. Selected Best Hooks are highlighted in bold. Results for C. intestinalis include those obtained with default and custom values (see main text).

Additional file 4: Supplementary Table 4. Putative SLS loci identified by SLFinder in the C. elegans dataset. Previously reported SL-RNAs are also indicated.

Additional file 5: Supplementary Figure 1. Common issues to consider when utilizing SLFinder.

Additional file 6: Supplementary Figure 2. Non-functional SL loci found during SLFinder analyses.

Additional file 7: Supplementary Table 5. Putative SL loci identified by SLFinder in the C. intestinalis dataset. Annotated IncRNA and protein-coding genes closer than 100 pb are also indicated.

Additional file 8: Supplementary Table 6. Putative SL loci identified by SLFinder in the H. vulgaris dataset. Annotated protein-coding genes closer than $100 \mathrm{pb}$ are also indicated.

Additional file 9: Supplementary Figure 3. Sequences of putative SL identified in $H$. vulgaris dataset.

Additional file 10: Supplementary Table 7. Putative SL loci identified by SLFinder in the S. mansoni dataset using Wormbase's genome assembly as reference (WBPS). Annotated protein-coding genes closer than $100 \mathrm{bp}$ are indicated.

Additional file 11: Supplementary Table 8. Putative SL loci identified by SLFinder in the S. mansoni dataset using GeneDB's genome assembly as reference. Annotated protein-coding genes closer than 100 bp are indicated.

\section{Abbreviations}

coi: Consistency orientation index; Ef: Hook match at the End of the transcript in Forward orientation; Er: Hook match at the End of the transcript in Reverse orientation; NGS: Next Generation Sequencing; occ: Observation Count Cutoff; pSL: Putative Splice Leader; Sf: Hook match at the Start of the transcript in Forward orientation; SL: Spliced Leader; Sr: Hook match at the Start of the transcript in Reverse orientation; WBPS: Wormbase Parasite

\section{Acknowledgements}

J.C. is a recipient of a doctoral scholarship from Agencia Nacional de Investigación e Innovación (ANII), Uruguay. H.M., U.K. and A.I. are members of the Uruguayan National Researchers System (SNI), and PEDECIBA, Uruguay.

Availability and requirements

Project name: SLFinder

Project home page: https://github.com/LBC-Iriarte/SLFinder.git

Operating system(s): Linux

Programming language: BASH 
Other requirements: BLAST 2.6.0 or higher, cd-hit-est 4.7 or higher, Jellifish 2.2.6 or higher, MAFFT 7.307 or higher, Seqkit 0.10 .0 or higher, trimAl 1.2rev59, Trinity 2.8.3 or higher and Weblogos 3.6 .0 or higher,

License: Creative Commons Attribution License (CC BY 4.0).

Any restrictions to use by non-academics: None

\section{Authors' contributions}

J.C. and A.I. conceived of the presented work with support from U.K. and H.M.. J.C., U.K. and A.I. designed the experiments. J.C. and H.J. performed the bioinformatics studies, software's Manual editing and tests. J.C., H.J., U.K., H.M. and A.I. analyzed and interpreted the results. J.C., U.K. and A.I. wrote the manuscript with support from H.M. All the authors discussed the results and commented on the manuscript. The author(s) read and approved the final manuscript.

\section{Funding}

This work was supported by grant FCE_3_2016_1_125297 to A.I. from Agencia Nacional de Investigación e Innovación (ANII), Uruguay. The funding agency played no role in the design of the study, analysis, interpretation of data, or in writing the manuscript.

\section{Ethics approval and consent to participate}

Not Applicable.

\section{Consent for publication}

Not Applicable.

\section{Competing interests}

The authors declare that they have no competing interests.

\section{Author details}

'Laboratorio de Biología Computacional, Departamento de Desarrollo Biotecnológico, Instituto de Higiene, Facultad de Medicina, Universidad de la República, Montevideo, Uruguay. ${ }^{2}$ Unidad de Genómica Evolutiva, Facultad de Ciencias, Universidad de la República, Montevideo, Uruguay. ${ }^{3}$ Sección Biología Celular, Facultad de Ciencias, Universidad de la República, Montevideo, Uruguay.

Received: 16 January 2020 Accepted: 17 June 2020

Published online: 08 July 2020

\section{References}

1. Hastings KEM. SL trans-splicing: easy come or easy go? Trends Genet. 2005;21:240-7.

2. Stricklin SL. C. elegans noncoding RNA genes. In: WormBook; 2005. https://doi.org/10.1895/wormbook.1.1.1.

3. Ganot P, Kallesøe T, Reinhardt R, Chourrout D, Thompson EM. Spliced-leader RNA trans splicing in a chordate, Oikopleura dioica, with a compact genome. Mol Cell Biol. 2004;24:7795-805.

4. Matsuo M, Katahata A, Satoh S, Matsuzaki M. Characterization of spliced leader trans-splicing in a photosynthetic rhizarian amoeba, Paulinella micropora, and its possible role in functional gene transfer. PLoS One. 2018;13:e0200961.

5. Bitar M, Boroni M, Macedo AM, Machado CR, Franco GR. The spliced leader trans-splicing mechanism in different organisms: molecular details and possible biological roles. Front Genet. 2013, 199;4(October). https://doi.org/10.3389/ fgene.2013.00199.

6. Matsumoto J, Dewar K, Wasserscheid J, Matsumoto J, Dewar K, Wasserscheid J, et al. High-throughput sequence analysis of Ciona intestinalis SL trans-spliced mRNAs: alternative expression modes and gene function correlates. Genome Res. 2010;20:636-45.

7. de Moraes Mourão M, Bitar M, Pereira Lobo F, Paula Peconick A, Grynberg P, Prosdocimi F, et al. A directed approach for the identification of transcripts harbouring the spliced leader sequence and the effect of trans-splicing knockdown in Schistosoma mansoni. Mem Inst Oswaldo Cruz. 2013;108:707-17.

8. Pettitt J, Harrison N, Stansfield I, Connolly B, Müller B. The evolution of spliced leader trans-splicing in nematodes. Biochem Soc Trans. 2010;38:1125-30. https://doi.org/10.1042/BST0381125.

9. Pettitt J, Philippe L, Sarkar D, Johnston C, Gothe HJ, Massie D, et al. Operons are a conserved feature of nematode genomes. Genetics. 2014;197:1201-11.

10. Agorio A, Chalar C, Cardozo S, Salinas G. Alternative mRNAs arising from trans-splicing code for mitochondrial and cytosolic variants of Echinococcus granulosus thioredoxin glutathione reductase. J Biol Chem. 2003;278:12920-8.

11. Boroni M, Sammeth M, Gava SG, Jorge NAN, MacEdo AM, MacHado CR, et al. Landscape of the spliced leader transsplicing mechanism in Schistosoma mansoni. Sci Rep. 2018:8:3877.

12. Rossia A, Jackb EJRA, Alvarado AS. Molecular cloning and characterization of SL3: a stem cell- specific SL RNA from the planarian Schmidtea mediterranea. Gene. 2014;533:156-67.

13. Philippe L, Pandarakalam GC, Fasimoye R, Harrison N, Connolly B, Pettitt J, et al. An in vivo genetic screen for genes involved in spliced leader trans-splicing indicates a crucial role for continuous de novo spliced leader RNP assembly. Nucleic Acids Res. 2017;45(14):8474-83.

14. Lasda EL, Blumenthal T. Trans-splicing. Wiley Interdiscip Rev RNA. 2011;2:417-34

15. Sather S, Agabian N. A 5' spliced leader is added in trans to both alpha- and beta-tubulin transcripts in Trypanosoma brucei. Proc Natl Acad Sci U S A. 1985;82:5695-9. https://doi.org/10.1073/pnas.82.17.5695.

16. Tessier L, Keller M, Chan RL, Fournier R, Weil J. Short leader sequences may be transferred from small RNAs to premature mRNAs by trans-splicing in Euglena. EMBO J. 1991;10:2621-5.

17. Brehm $\mathrm{K}$, Jensen $\mathrm{K}$, Frosch M. mRNA trans-splicing in the human parasitic cestode Echinococcus multilocularis. J Biol Chem. 2000;275:38311-8. 
18. Rajkovic A, Davis RE, Simonsen JN, Rottman FM. A spliced leader is present on a subset of mRNAs from the human parasite Schistosoma mansoni. Proc Natl Acad Sci U S A. 1990;87:8879-83. https://doi.org/10.1073/pnas.87.22.8879.

19. Krause M, Hirsh D. A trans-spliced leader sequence on actin mRNA in C. elegans. Cell. 1987;49:753-61.

20. Ross LH, Freedman JH, Rubin CS. Structure and expression of novel spliced leader RNA genes in Caenorhabditis elegans. J Biol Chem. 1995;270:22066-75 http://www.ncbi.nlm.nih.gov/pubmed/7665629.

21. Vandenberghe AE, Meedel TH, Hastings KEM. mRNA 5'-leader trans-splicing in the chordates. Genes Dev. 2001;15:294-303.

22. Pouchkina-Stantcheva NN, Tunnacliffe A. Spliced leader RNA-mediated trans-splicing in phylum rotifera. Mol Biol Evol. 2005:22:1482-9.

23. Stover NA, Steele RE. Trans-spliced leader addition to mRNAs in a cnidarian. Proc Natl Acad Sci. 2001;98:5693-8. https:// doi.org/10.1073/pnas.101049998

24. Lidie KB, Van Dolah FM. Spliced leader RNA-mediated trans-splicing in a dinoflagellate, Karenia brevis. J Eukaryot Microbiol. 2007;54:427-35.

25. Douris $\mathrm{V}$, Telford MJ, Averof M. Evidence for multiple independent origins of trans-splicing in Metazoa. Mol Biol Evol. 2010;27:684-93.

26. Lei Q, Li C, Zuo Z, Huang C, Cheng H, Zhou R. Evolutionary insights into RNA trans-splicing in vertebrates. Genome Biol Evol. 2016:8:562-77.

27. Nilsson D, Gunasekera K, Mani J, Osteras M, Farinelli L, Baerlocher L, et al. Spliced leader trapping reveals widespread alternative splicing patterns in the highly dynamic transcriptome of Trypanosoma brucei. PLoS Pathog. 2010;6:e1001037.

28. Cuypers B, Domagalska MA, Meysman P, De Muylder G, Vanaerschot M, Imamura H, et al. Multiplexed spliced-leader sequencing: a high-throughput, selective method for RNA-seq in Trypanosomatids. Sci Rep. 2017;7:0-11..

29. Gopal S, Awadalla S, Gaasterland T, Cross GAM. A computational investigation of kinetoplastid trans-splicing. Genome Biol. 2005;6:R95.

30. Kelly S, Wickstead B, Maini PK, Gull K. Ab initio identification of novel regulatory elements in the genome of Trypanosoma brucei by Bayesian inference on sequence segmentation. PLoS One. 2011;6:e25666.

31. Barnes SN, Masonbrink RE, Maier TR, Seetharam A, Sindhu AS, Severin AJ, et al. Heterodera glycines utilizes promiscuous spliced leaders and demonstrates a unique preference for a species-specific spliced leader over C. elegans SL1. Sci Rep. 2019:67:1356. https://doi.org/10.1038/s41598-018-37857-0.

32. Fiebig M, Gluenz E, Carrington M, Kelly S. Molecular \& biochemical parasitology SLaP mapper: a webserver for identifying and quantifying spliced-leader addition and polyadenylation site usage in kinetoplastid genomes. Mol Biochem Parasitol. 2014;196:71-4. https://doi.org/10.1016/j.molbiopara.2014.07.012.

33. Radío S, Fort RS, Garat B, Sotelo-silveira J. UTRme: a scoring-based tool to annotate untranslated regions in trypanosomatid genomes. Front Genet. 2018;9:671.

34. Yague-sanz C, Hermand D. SL-quant: a fast and flexible pipeline to quantify spliced leader trans-splicing events from RNA-seq data. Gigascience. 2018;7:1-7.

35. Conesa A, Madrigal P, Tarazona S, Gomez-Cabrero D, Cervera A, McPherson A, et al. A survey of best practices for RNAseq data analysis. Genome Biol. 2016;17:13. https://doi.org/10.1186/s13059-016-0881-8.

36. Chapman JA, Kirkness EF, Simakov O, Hampson SE, Mitros T, Weinmaier T, et al. The dynamic genome of Hydra. Nature. 2010;464:592-6.

37. Pettitt J, Mu B, Stansfield I, Connolly B. Spliced leader trans-splicing in the nematode Trichinella spiralis uses highly polymorphic, noncanonical spliced leaders. RNA. 2008:14:760-70.

38. van Bers NEM. Characterization of genes coding for small hypervariable peptides in Globodera rostochiensis: Wageningen University; 2008. http://edepot.wur.nl/16343.

39. Guo Y, Bird DM, Nielsen DM. Improved structural annotation of protein-coding genes in the Meloidogyne hapla genome using RNA-Seq. Worm. 2014;16:e29158.

40. Roy SW. Genomic and Transcriptomic analysis reveals spliced leader trans-splicing in Cryptomonads. Genome Biol Evol. 2017:9:468-73

41. Tsai IJ, Zarowiecki M, Holroyd N, Garciarrubio A, Sanchez-Flores A, Brooks KL, et al. The genomes of four tapeworm species reveal adaptations to parasitism. Nature. 2013;496:57-63.

42. Wasik K, Gurtowski J, Zhou X, Ramos OM, Delás MJ, Battistoni G, et al. Genome and transcriptome of the regenerationcompetent flatworm, Macrostomum lignano. Proc Natl Acad Sci. 2015;112:12462-7. https://doi.org/10.1073/pnas.1516718112.

43. Yang F, Xu D, Zhuang Y, Yi X, Huang Y, Chen $H$, et al. Spliced leader RNA trans-splicing discovered in copepods. Sci Rep. 2015;5:17411. https://doi.org/10.1038/srep17411.

44. Zhang H, Dungan CF, Lin S. Introns, alternative splicing, spliced leader trans-splicing and differential expression of pcna and cyclin in Perkinsus marinus. Protist. 2011;162:154-67.

45. Grabher MG, Haas BJ, Yassour M, Levin JZ, Thompson DA, Amit I, et al. Trinity: reconstructing a full-length transcriptome without a genome from RNA-Seq data. Nat Biotechnol. 2013;29:644-52.

46. Haas BJ, Papanicolaou A, Yassour M, Grabherr M, Blood PD, Bowden J, et al. De novo transcript sequence reconstruction from RNA-seq using the trinity platform for reference generation and analysis. Nat Protoc. 2013:8:1494-512. https://doi. org/10.1038/nprot.2013.084

47. Marçais G, Kingsford C. A fast, lock-free approach for efficient parallel counting of occurrences of k-mers. Bioinformatics. 2011;27:764-70.

48. Altschul SF, Gish W, Miller W, Myers EW, Lipman DJ. Basic local alignment search tool. J Mol Biol. 1990;215:403-10. https://doi.org/10.1016/S0022-2836(05)80360-2.

49. Yang Y, Smith SA. Optimizing de novo assembly of short-read RNA-seq data for phylogenomics. BMC Genomics. 2013; 14:328.

50. Wang S, Gribskov M. Comprehensive evaluation of de novo transcriptome assembly programs and their effects on differential gene expression analysis. Bioinformatics. 2017;33:327-33.

51. Fu L, Niu B, Zhu Z, Wu S, Li W. CD-HIT: accelerated for clustering the next-generation sequencing data. Bioinformatics. 2012;28:3150-2

52. Katoh K, Standley DM. MAFFT multiple sequence alignment software version 7: improvements in performance and usability. Mol Biol Evol. 2013:30:772-80 
53. Capella-gutiérrez S, Silla-martínez JM, Gabaldón T. trimAl: a tool for automated alignment trimming in large-scale phylogenetic analyses. Bioinformatics. 2009;25:1972-3.

54. Crooks GE, Hon G, Chandonia J, Brenner SE. WebLogo: a sequence logo generator. Genome Res. 2004;14:1188-90.

55. Protasio AV, Tsai IJ, Babbage A, Nichol S, Hunt M, Aslett MA, et al. A systematically improved high quality genome and transcriptome of the human blood fluke Schistosoma mansoni. PLoS Negl Trop Dis. 2012;6:e1455.

56. Andrews S. FastQC: a quality control tool for high throughput sequence data. 2010. https:/www.bioinformatics. babraham.ac.uk/projects/fastqc/. Accessed 6 June 2018.

57. Bolger AM, Lohse M, Usadel B, Planck M, Plant M, Mühlenberg A. Trimmomatic: a flexible trimmer for Illumina sequence data. Bioinformatics. 2014:30:2114-20.

58. Johns Hopkins University, Center for Computational Biology. GFF utilities. http://ccb.jhu.edu/software/stringtie/gff.shtml. Accessed 6 June 2018.

59. Hall TA. BioEdit: a user-friendly biological sequence alignment editor and analysis program for Windows 95/98/NT. Nucleic Acids Symp. 1990;41:95-8.

60. Tan G, Muffato M, Ledergerber C, Herrero J, Goldman N, Gil M, et al. Current methods for automated filtering of multiple sequence alignments frequently worsen single-gene phylogenetic inference. Syst Biol. 2015;64:778-91.

61. WBPS14. Schistosoma mansoni. 2019. https://parasite.wormbase.org/Schistosoma_mansoni_prjea36577/Info/Index/. Accessed 11 Dec 2019

62. Hampson S, Kibler D, Baldi P. Distribution patterns of over-represented k-mers in non-coding yeast DNA. Bioinformatics. 2002;18:513-28.

Publisher's Note

Springer Nature remains neutral with regard to jurisdictional claims in published maps and institutional affiliations.

Ready to submit your research? Choose BMC and benefit from:

- fast, convenient online submission

- thorough peer review by experienced researchers in your field

- rapid publication on acceptance

- support for research data, including large and complex data types

- gold Open Access which fosters wider collaboration and increased citations

- maximum visibility for your research: over $100 \mathrm{M}$ website views per year

At $\mathrm{BMC}$, research is always in progress.

Learn more biomedcentral.com/submissions 